$\mathrm{SCIDOC}$
International Journal of Dentistry and Oral Science (IJDOS)

ISSN: $2377-8075$

\title{
Comparison of Infrazygomatic Crest Mini-Implant and Inter-Radicular Mini-Implant as Skeletal Anchorage when used for En Masse Anterior Retraction and En Masse Distalization - A Fem Study
}

Research Article

Swapna Sreenivasagan ${ }^{1}$, Aravind Kumar Subramanian ${ }^{2 *}$, Jong-Moon Chae ${ }^{3}$, Stephen Mark Chadwick ${ }^{4}$

${ }^{1}$ Resident, Department of Orthodontics, Saveetha Dental College, Saveetha Institute of Medical and Technical Science, Saveetha University, Chennai, India.

${ }^{2}$ Professor and Head of the Department, Department of Orthodontics, Saveetha Dental College, Saveetha Institute of Medical and Technical Science, Saveetha University, Chennai, India.

${ }^{3}$ Professor, Department of Orthodontics, School of Dentistry, University of Wonkwang, Wonkwang Dental Research Institute, Iksan, Korea; Visiting Scholar, Postgraduate Orthodontic Program, Arizona School of Dentistry and Oral Health, A.T. Still University, Mesa, Ariz.

${ }^{4}$ Professor and Consultant orthodontist, NHS Royal College of Physicians and Surgeons of Glasgow,London, United Kingdom.

\section{Abstract}

Introduction: This paper describes a finite element model study comparing infrazygomatic crest mini-implants with inter-radicular mini-implants for both En Masse retraction of anterior teeth and En masse distalization of the upper dentition.

Aim: The main objectives of this study were to examine the forces on the bone generated by the alternative biomechanic systems of anterior retraction or en masse distalization.

Design: Finite element analysis.

Setting: Department of orthodontics.

Material and Methods: Finite element models of maxillary anterior teeth and surrounding bone were constructed from CT scans Main outcome methods: FEM model was used to simulate the force levels and direction using the two alternative mini-implant systems under investigation.

Results: There was no significant difference between the force levels used in infrazygomatic crest mini-implants compared to inter-radicular mini-implants. Maximum distalization was achieved when the infrazygomatic crest mini-implants were placed at 70 degrees. The infrazygomatic crest mini-implants were more effective in en masse distalization of the maxillary dentition. If both intrusion and retraction are indicated infrazygomatic mini-implants are a better choice than inter-radicular mini-implants.

Conclusions: The infrazygomatic crest mini-implants were more effective in en masse distalization of the maxillary dentition. If both intrusion and retraction are indicated infrazygomatic mini-implants are a better choice than inter-radicular mini-implants.

Keywords: Finite Element Analysis; Intrusion; Retraction; Infrazygomatic Crest Mini-Implant; Inter-Radicular Mini-Implant.

\section{Introduction}

Anchorage has been described as the resistance to unwanted tooth movement. As many of our patients present with proclined upper anterior teeth and an increased overjet it is desirable for these teeth to be retroclined and retracted. The reaction to this desirable tooth movement is the undesirable mesial movement of the upper posterior molars. As a consequence anchorage has often involved headgear, palatal arches or temporary anchorage devices in maxilla in order to resist this undesirable mesial movement of the upper molar teeth. Our paper goes further than preventing the upper molar teeth moving mesially as a reaction to the retraction of the upper incisors and looks at the En Masse distalization of the whole of the maxillary dentition. This is a new development for our speciality as this type of En Masse movement of the whole dentition was previously only possible using orthognathic surgery.

\section{*Corresponding Author:}

Dr. Aravind Kumar Subramanian,

Professor and Head of the Department, Department of Orthodontics, Saveetha Dental College, Saveetha Institute of Medical and Technical Science, Saveetha University, Chennai, India.

Tel: +919841299939

E-mail: aravindkumar@saveetha.com

Received: November 01, 2020

Accepted: December 03, 2020

Published: December 04, 2020

Citation: Swapna Sreenivasagan, Aravind Kumar Subramanian, Jong-Moon Chae, Stephen Mark Chadwick. Comparison of Infrazygomatic Crest Mini-Implant and Inter-Radicular Mini-Implant as Skeletal Anchorage when used for En Masse Anterior Retraction and En Masse Distalization - A Fem Study. Int J Dentistry Oral Sci. 2020;7(12):1176-1181. doi: http://dx.doi.org/10.19070/2377-8075-20000233

Copyright: Aravind Kumar Subramanian ${ }^{\circ} 2020$. This is an open-access article distributed under the terms of the Creative Commons Attribution License, which permits unrestricted use, distribution and reproduction in any medium, provided the original author and source are credited. 
Stable molar anchorage is a pre-requisite for orthodontic treatment with fixed appliances [1]. Skeletal anchorage with mini-implants has been reported as an adjunct to orthodontic treatment that is less dependent on patient compliance [2]. In split mouth studies comparing conventional anchorage and mini-implants retraction can be achieved with no loss of anchorage on the implant side whilst on on the non implant side there will be a small loss of anchorage [3]. Mini-implants can be an efficient alternative to the conventional molar anchorage especially in maximum anchorage situations [1]. Not only have these screws proved to be useful for anchorage in extraction cases, they have also been used cases where it is desirable to distallize the entire arch [4].

The finite element method is a widely used mathematical model for solving problems in engineering. It has been used in structural analysis, heat transfer, mass transport and electromagnetic potential. Finite element analysis is a way of mathematically modelling the stresses on an engineering design. Finite element analysis has found increasing use in the field of orthodontics due to its ability to deliver detailed yet precise information regarding stress on load application [5]. Finite element method (FEM) studies can be useful for us to understand the various factors that we can't clinically assess.

The variability of material characteristics, the loads applied to the orthodontic appliance and the dentition, the stress patterns and their geometric evaluation [6]. Using the finite element method complex structures like the maxilla are divided into smaller units called finite elements which helps in formulating a solution for each element rather than for the whole arch [7]. Computational techniques have helped our understanding of biomechanics.

Finite element method has been helpful in orthodontics as it is impractical to use randomized clinical trials to compare every modification to biomechanics or to appliance design. Finite element method have been used to simulate the addition of miniimplants, the addition of power arms to brackets, the intrusion and extrusion of teeth and variations in the arch wire dimensions $[7-10]$.

Infrazygomatic crest mini-implants are usually made of either stainless steel or titanium and placed in the region of infrazygomatic crest distal to the first permanentmolar. They are used mostly in en masse distalization and in orthodontic correction of malocclusions without surgery or extractions [11]. The distal movement of anterior teeth in conventional mechanics is usually inefficient and slow. Where as mini-screws offers the practitioner the opportunity to translate the entire anterior quadrant. Thereby reducing the treatment time, eliminating any loss of anchorage and also reducing patient discomfort [12] Miniscrews have been used in non-extraction cases and maintained throughout treatment in order to provide anchorage for the retraction of the whole dentition [13].

The main objective of this paper is to evaluate the en masse retraction of the anterior teeth and the en masse distalization of the maxillary dentition. Using infrazygomatic crest mini-implants or interradicular mini-implants. The outcome measures are bone stress, the amount of retraction and the amount of intrusion of maxillary teeth.

\section{Methodology}

CT scans of human skulls were obtained in DICOM format. These images were then changed to STL images using MIMICS 8.11 software. The design of mini-implant used in this model was obtained from Favanchor company, the inter-radicular mini-implant was of $1.6^{*} 10 \mathrm{~mm}$ in size and infrazygomatic mini-implant of size $2 * 12 \mathrm{~mm}$. Using these geometric models of lines and surfaces were made and surface model of a finite element model tool was using HYPERMESH software version 10.0. ABAQUS software was used for material design. The hinge portion of maxilla was fixed in all degrees of freedom.

Once the maxilla model was generated the upper teeth were set up with metal brackets of MBT system and all tooth aligned, with wire of $19 * 25$ dimension stainless steel (Figure 1). For the model to be used for en masse anterior retraction, the first premolar was removed from both the sides. (Figure 2) En masse distalization setup was done and brackets placed till the second molars (Figure 3).

The infra-zygomatic crest mini-implant were placed between the 1 st and 2 nd molar $13 \mathrm{~mm}$ above the CEJ at 5 different angulation of $50,60,70,80$ and $90^{\circ}$ from the level of the occlusal plane. The orientation perpendicular to the buccal bone was taken as $90^{\circ}$ and reduction of 10 degrees [11].

The interradicular mini-implant was placed between the tooth roots of 2 nd premolar and $1 \mathrm{st}$ molar at $7 \mathrm{~mm}$ above the CEJ at 3 different angulations of 30,40 and $50^{\circ}$ from the level of the occlusal plane. The miniscrews placed in maxilla are usually placed at $30-40^{\circ}$ to the long axis of the posterior teeth and whereas in mandible 10-20 degrees of angulation in the mandible.

Anteriorly retraction hooks of $8 \mathrm{~mm}$ length were placed between the lateral incisor and canine. For the en masse anterior retraction model the force applied on either side was fixed at $200 \mathrm{gms}$ per side and en masse distalization model 300 gms per side using niti coil springs. This model of maxilla was fixed in all directions and discretized in $\mathrm{X}, \mathrm{Y}$ and $\mathrm{Z}$ axis.

\section{Results}

The properties of the materials used in this FEM set-up has been depicted in young's modulus and as poisson's ratio in Table 1 and they were assumed to be isotropic and homogenous.

Table 2 depicts the results of Von mises test, the bone stress patterns when infrazygomatic crest mini-implants and interradicular mini-implants when used for en masse distalization and en masse retraction. Maximum bone stress is noted when the angulation was reduced in IZC mini-implants. There was no significant difference in the stress levels when mini-implants were tried with different angulations. Figure 4 is a depiction of von mises test.

The magnitude of backward movement or the retraction of anterior teeth is shown in Table 3, the infrazygomatic crest miniimplant at 70 degrees brought about maximum movement when used for both the en masse distalization model ad en masse anterior retraction model whereas there is no significant difference in the movement between the various angles tried for inter-radicular 
Table 1. Material properties.

\begin{tabular}{|c|c|c|c|c|c|c|}
\hline \multicolumn{7}{|c|}{ Table of Material properties } \\
\hline Name & $\begin{array}{c}\text { Titanium } \\
\text { implant }\end{array}$ & $\begin{array}{c}\text { Stainless steel } \\
\text { implant }\end{array}$ & $\begin{array}{c}\text { Maxilla } \\
\text { Bone }\end{array}$ & Teeth & Arch wire & Bracket \\
\hline $\begin{array}{c}\text { Young's Modulus } \\
\text { (Mpa) }\end{array}$ & $1,13,000$ & 200000 & 200 & 20000 & 200000 & 200000 \\
\hline Poisson's ratio & 0.3 & 0.3 & 0.3 & 0.3 & 0.3 & 0.3 \\
\hline
\end{tabular}

Table 2. Bone Stress associated infrazygomatic crest mini-implants and inter-radicular crest mini-implants.

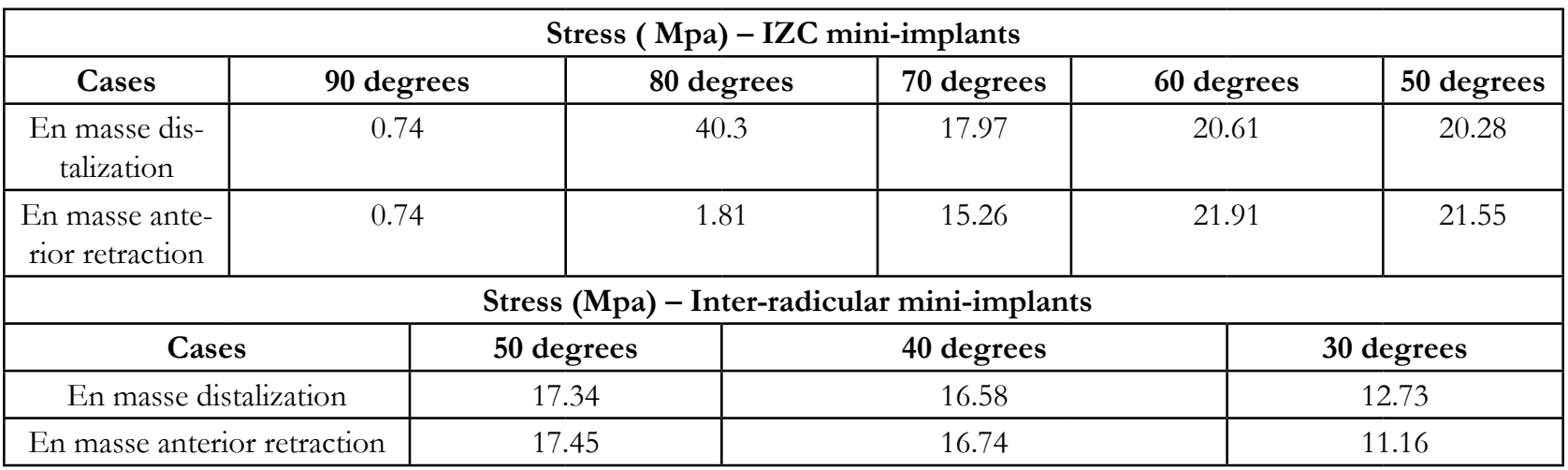

Table 3. Displacement in Backward direction using Infrazygomatic crest mini-implant and inter-radicular crest mini-implants.

Backward displacement in microns

\begin{tabular}{|c|c|c|c|c|c|}
\hline IZC & 90 degrees & 80 degrees & 70 degrees & 60 degrees & 50 degrees \\
\hline En masse distalization & 1.34 & 2.7 & 8.58 & 7.97 & 7.84 \\
\hline En masse anterior retraction & 1.28 & 1.07 & 11.5 & 10.2 & 10 \\
\hline
\end{tabular}

Backward displacement in microns

\begin{tabular}{|c|c|c|c|c|c|}
\hline Inter-radicular mini-implants & $\mathbf{5 0}$ degrees & 40 degrees & 30 degrees & & \\
\hline En masse distalization & 6.75 & 6.74 & 6.82 & & \\
\hline En masse anterior retraction & 8.72 & 8.91 & 9.08 & & \\
\hline
\end{tabular}

Table 4. Intrusion tooth movement when using Infrazygomatic crest mini-implant and inter-radicular crest mini-implants.

\begin{tabular}{|c|c|c|c|c|c|}
\hline IZC & 90 degrees & 80 degrees & 70 degrees & 60 degrees & 50 degrees \\
\hline En masse distalization & 1.34 & 1.29 & 9.64 & 9.41 & 9.13 \\
\hline En masse anterior retraction & 1.35 & 1.16 & 8.39 & 8.36 & 8.1 \\
\hline Inter-radicular mini-implants & \multicolumn{2}{|c|}{50 degrees } & 40 degrees & \multicolumn{2}{c|}{30 degrees } \\
\hline En masse distalization & \multicolumn{2}{|c|}{5.85} & 5.72 & \multicolumn{2}{c|}{5.21} \\
\hline En masse anterior retraction & \multicolumn{2}{|c|}{4.69} & 4.72 & \multicolumn{2}{c|}{4.33} \\
\hline
\end{tabular}

mini-implants. IZC mini-implants brought about more distalization when compared to inter-radicular mini-screws. Figure 5 is a depiction of the the results of the distal movements in magnitude. Table 4 is used to explain the movement in magnitude in the $\mathrm{z}$ direction or the intrusive movement . Maximum intrusive movement was noted when force was applied with IZC, the intrusive movement is almost negligible when seen with inter-radicular implants. Figure 6 explains the studies that revealed in displacement in intrusive movements.

\section{Discussion}

[14] One of the perceived complications of the retraction of upper anterior teeth using sliding mechanics is the extrusion of the upper anterior teeth during the retraction process. This would be a particular risk for patients presenting with a high smile line or vertical maxillary excess [14]. The use of mini-implants can reduce this side effect.Miniscrews in posterior maxilla are used to bring about various tooth movement mainly the retraction of anterior teeth and the distalization of all the teeth [15]. The or- 
Figure 1. Model with brackets , $19 * 25$ ss wire and mini-implants ,with force applied.

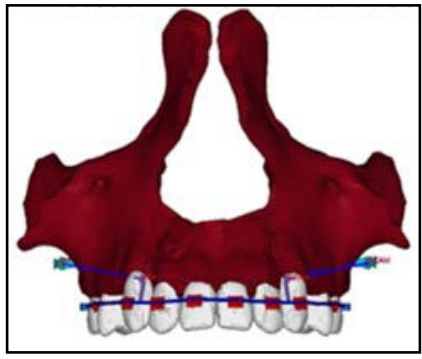

Figure 2. Model for anterior retraction.

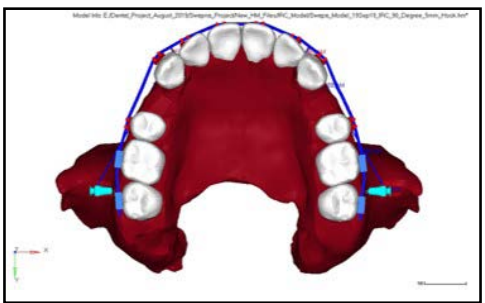

Figure 3. Model for En masse distalization.

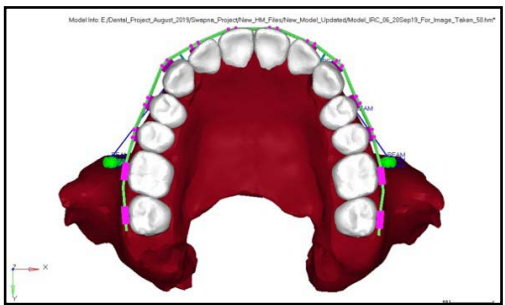

Figure 4. Von mises test results on bone stress patterns.

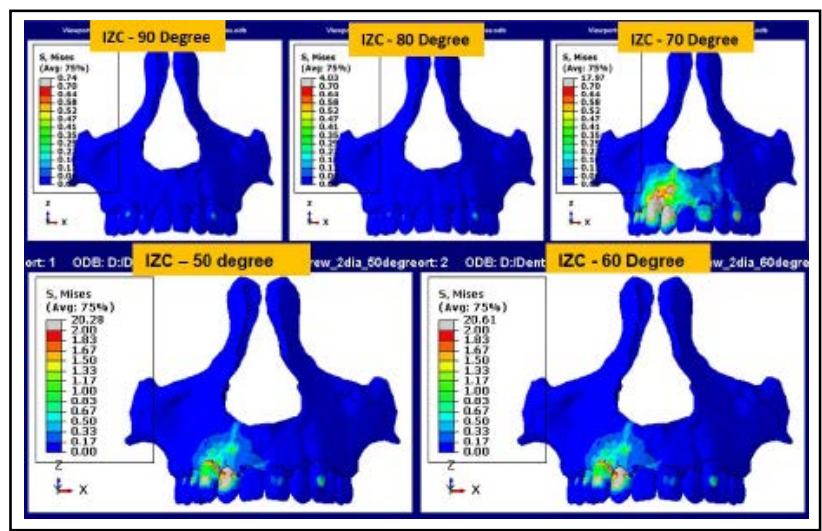

Figure 5. Displacement in magnitude -retraction.

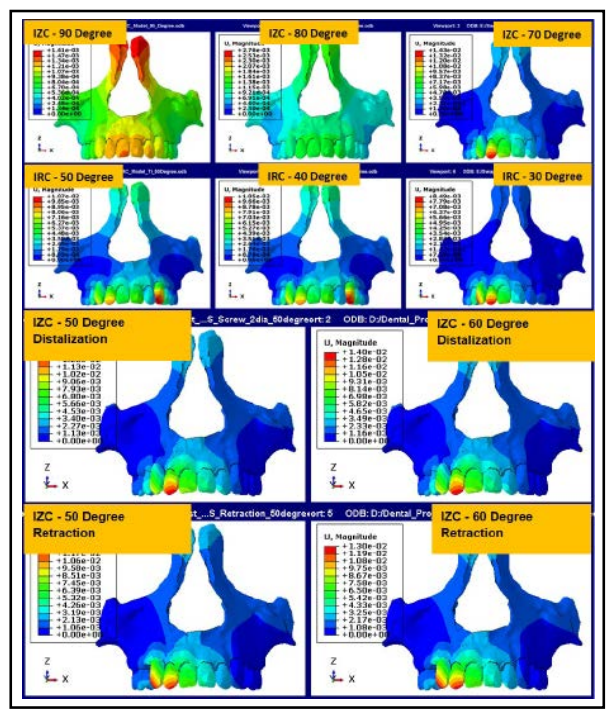


Figure 6. Displacement -intrusive movement.

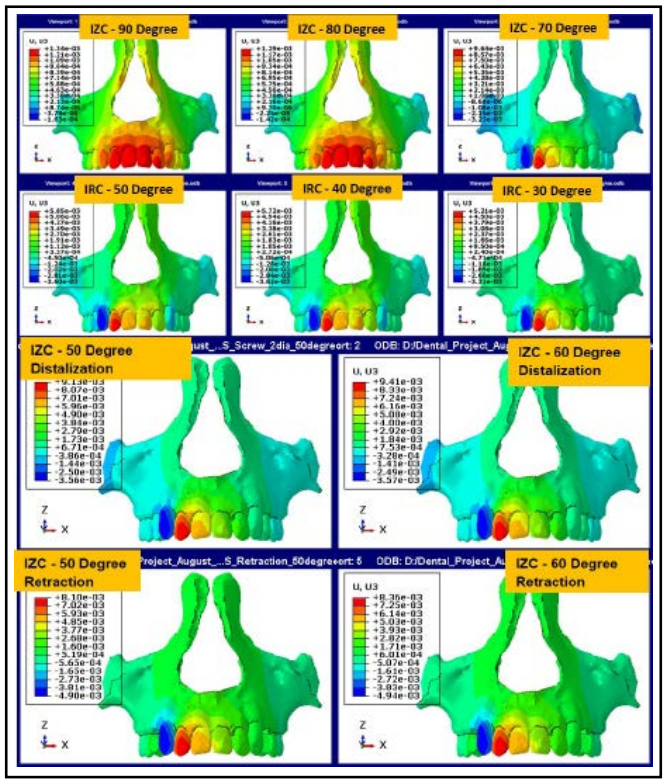

thodontic mini implants can be useful not only as a skeletal anchorage, but also for torque control during retraction [16]. The true retraction will take place when the force passes through the centre of resistance of the anterior teeth. When mini-screws are placed between the second premolar and the first molar there is increase in rotation of anterior teeth and the vertical movement is reduced. For en masse distalization infrazygomatic crest miniimplants are mostly indicated however it has been proposed that interradicular implants can be used for the same effect [5].

There are various studies both in-vitro and in FEM models where mini-implants have been widely researched in order to evaluate the results on retraction of anterior teeth and for en masse anterior retraction. This study is the first of its kind to evaluate and compare the infrazygomatic crest mini-implants and the interradicular mini-implants and to gain an understanding of the bone stress patterns This study also aimed at establishing which miniimplant was better for the purpose of en masse retraction of the anterior teeth and for the en masse distalization of all the teeth of the maxilla. The main change due to the orthodontic treatment is due to tissue reaction either through bone or within the bone, hence it is essential to study the stress patterns [15].

From the results of our study there is greater bone stress when infra zygomatic crest mini-implants are placed at 70 degrees and when interradicular mini-implants are placed at 40 and 50 degrees.

There is no significant difference between Infrazygomatic implant and interradicular mini-implant in bringing about anterior en masse anterior retraction. However, infra zygomatic crest mini-implants is better for en masse distalization of the maxillary dentition.

Using Interradicular mini-implant there is no significant intrusion.

The mini-implants placed at an increase in the acute angulation has a potential to engage more cortical bone thickness, there is also a disadvantage that too acute an angulation will cause slippage during placement [16] Liou has established that insertion angle of 30 to 70 degrees from the occlusal plane is sufficient to allow adequate cortical bone engagement and more success of the mini-implants which is in accordance with the results of our study [17].

In a previous study conducted there was no significant level of difference in stress and magnitude when comparing the titanium and stainless steel mini-screws. In the present study we have used titanium mini-screw in both the regions in order to eliminate bias that can arise from different material properties.

FEM is only an approximation technique and are highly dependent on the models generated. There is an inability to predict long term effects and movement and stress distribution after force application. Time dependant reactions are unpredictable and needs clinical evidence.

\section{Conclusion}

For En masse distalization infrazygomatic crest mini-implants are more efficient than inter-radicular mini-implants.

There is no significant difference between these two alternatives for en masse retraction of the upper anterior teeth.

Infrazygomatic crest mini-implants are indicated when both retraction and intrusion of the teeth is desirable.

\section{Acknowledgements}

The researchers didn't receive any funding from external sources for this project. We sincerely thank engineer Mr Ramkumar. M for helping in the finite element model setup and analysis.

\section{References}

[1]. Yamaguchi M, Inami T, Ito K, Kasai K, Tanimoto Y. Mini-Implants in the Anchorage Armamentarium: New Paradigms in the Orthodontics. International Journal of Biomaterials. 2012; 2012: 394121. Pubmed PMID: 22719763.

[2]. Costa A, Pasta G, Bergamaschi G. Intraoral hard and soft tissue depths for temporary anchorage devices. Seminars in Orthodontics. 2005 Mar 1; 11(1): 10-5. 
[3]. Thiruvenkatachari B, Pavithranand A, Rajasigamani K, Kyung HM. Comparison and measurement of the amount of anchorage loss of the molars with and without the use of implant anchorage during canine retraction. American Journal of Orthodontics and Dentofacial Orthopedics. 2006 Apr 1;129(4):551-4. Pubmed PMID: 16627183.

[4]. Chung KR, Choo H, Kim SH, Ngan P. Timely relocation of mini-implants for uninterrupted full-arch distalization. American journal of orthodontics and dentofacial orthopedics. 2010; 138(6): 839-849. Pubmed PMID: 21130344 .

[5]. Marya A. Finite Element Analysis and its Role in Orthodontics. Advances in Dentistry \& Oral Health. 2016 Jul 28; 2: 5-6.

[6]. CicciùM, BramantiE, CecchettiF, ScappaticciL, GuglielminoE, RisitanoG. Fem and Von Mises analyses of different dental implant shapes for masticatory loading distribution. Oral Implantol (Rome). 2014 Dec 27; 7(1): 1-10. Pubmed PMID: 25694795.

[7]. Sung SJ, Jang GW, Chun YS, Moon YS. Effective en-masse retraction design with orthodontic mini-implant anchorage: A finite element analysis. American Journal of Orthodontics and Dentofacial Orthopedics. 2010 May 1; 137(5): 648-57. Pubmed PMID: 20451784.

[8]. Hedayati Z, Hashemi SM, Zamiri B, Fattahi HR. Anchorage value of surgical titanium screws in orthodontic tooth movement. International Journal of Oral and Maxillofacial Surgery. 2007 Jul 1; 36(7): 588-92. Pubmed PMID: 17524619 .

[9]. Kim T, Suh J, Kim N, Lee M. Optimum conditions for parallel translation of maxillary anterior teeth under retraction force determined with the finite element method. American Journal of Orthodontics and Dentofacial Orthopedics. 2010 May 1;137(5):639-47. Pubmed PMID: 20451783.

[10]. Ahuja S, Gupta S, Bhambri E, Ahuja V, Jaura BS. Comparison of conven- tional methods of simultaneous intrusion and retraction of maxillary anterior: a finite element analysis. Journal of Orthodontics. 2018 Oct 2; 45(4): 243-9. Pubmed PMID: 302806645.

[11]. Chang C, Liu SSY, Roberts WE. Primary failure rate for 1680 extra-alveolar mandibular buccal shelf mini-screws placed in movable mucosa or attached gingiva. The Angle Orthodontist. 2015 Jan 20; 85(6): 905-10. Pubmed PMID: 25603272.

[12]. Park HS, Jeong SH, Kwon OW. Factors affecting the clinical success of screw implants used as orthodontic anchorage. American Journal of Orthodontics and Dentofacial Orthopedics. 2006 Jul 1; 130(1): 18-25. Pubmed PMID: 16849067.

[13]. Park HS, Kwon TG, Sung JH. Nonextraction Treatment with Microscrew Implants. The Angle Orthodontist. 2004 Aug 1;74(4): 539-49. Pubmed PMID: 15387034.

[14]. Jeong GM, Sung SJ, Lee KJ, Chun YS, Mo SS. Finite-element investigation of the center of resistance of the maxillary dentition. Korean Journal of Orthodontics. 2009 Apr 1; 39(2): 83-94.

[15]. Melsen B. Biological reaction of alveolar bone to orthodontic tooth movement. The Angle Orthodontist. 1999 Apr 1; 69(2): 151-8. Pubmed PMID: 10227556.

[16]. Kravitz ND, Kusnoto B. Risks and complications of orthodontic miniscrews. American Journal of Orthodontics and Dentofacial Orthopedics. 2007 Apr 1; 131(4, Supplement): S43-51. Pubmed PMID: 17448385.

[17]. Liou EJW, Pai BCJ, Lin JCY. Do miniscrews remain stationary under orthodontic forces?. American Journal of Orthodontics and Dentofacial Orthopedics. 2004 Jul 1; 126(1): 42-7. Pubmed PMID: 15224057. 